\title{
Some significant trends in health care textiles
}

\begin{abstract}
The article highlights some recent trends in the development of health care textiles. The health care practices for medical textiles in government hospitals of certain countries of the world have been studied. Specifically, the study determined the availability and maintenance of medical textiles in government hospitals. There is a growing concern related to the effects of nanomaterials in health and safety. Nanotechnologies are already present in many consumer products, including textiles. "Nanotextiles" can be considered as traditional textiles with the incorporation of nanoparticles. They present often functionalities such as antibacterial, ultraviolet radiation protection, water and dirt repellency, self-cleaning or flame retardancy.
\end{abstract}

Keywords: medical textile, healthcare, nano materials, safety, traditional textiles, water, healthcare, urogynecologic slings, illness, health professionals, healthcare service, occupational safety, medical textiles, $\mathrm{x}$-ray clothes, pulmonary inflammation, genotoxicity, carcinogenicity
Volume 2 Issue 3 - 2018

\author{
N Gokarneshan \\ Department of Textile Technology, Park College of engineering \\ and technology Coimbatore, India
}

\author{
Correspondence: N Gokarneshan, Department of Textile \\ Technology, Professor and Head, Park College of engineering \\ and technology Coimbatore, India,
} Email advaitcbe@rediffmail.com

Received: April 30, 2018 | Published: June 13, 2018

\section{Introduction}

Healthcare is the service rendered by health professionals for the benefit of patients and could be inform of prevention, treatment and management of illness, the prevention of mental, problems and promotion of general well-being of man through the services offered by the medical and allied health professionals. ${ }^{1}$ The institute of Medicine defined healthcare as the health services provided to individuals and patients to improve desired health outcomes. ${ }^{2}$ Merriam noted that healthcare is the maintenance and restoration of health by professional in medicine, clinical psychology, dentistry and public health. ${ }^{3}$ Merriam maintained that the services of healthcare are typically offered to patients through a healthcare system made up of hospitals and health professionals. Healthcare is therefore, the treatment give to invalids in the hospitals and outside hospitals by trained medical personnel. Recently there has been a rapid emergence of nanotechnology into several consumer products, which has led to concerns as regards the potential risk for human health following consumer exposure. There is also a concern in terms of occupational safety and health, related to the exposure of workers involved in manufacturing, processing and handling of consumer goods containing nanomaterials. Nanosafety is a growing concern: exposure to engineered nanomaterials has been associated with a number of health effects including pulmonary inflammation, genotoxicity, carcinogenicity and circulatory effects. ${ }^{4}$

\section{Health care for medical textiles in hospitals}

Bircher noted that healthcare services are affected through the proper medical textiles technology by medical professionals. ${ }^{5}$ The implementation and progress of healthcare depends on the provision of healthcare services, equipment such as medical textiles x-ray clothes (lead suite) oxygen mask rubber and catheter. ${ }^{6}$ Medical textiles according to Impex are all textiles used in the operative and post operative tasks in and around patients and medical practitioners. ${ }^{7}$ Mazharul noted that medical textile is a branch of technical textiles that are usually made with specific care in collaboration with the hygienic manufacturing procedures. ${ }^{8}$ By implication medical textiles are therefore, the textiles that are specially made from both artificial and natural fibres which are used in hospitals and other healthcare sectors. Impex classified medical textile into five namely: knitted medical textiles, braided medical textiles, woven medical textiles, nonwoven medical textiles, and healthcare and hygiene product medical textiles. ${ }^{9}$ Knitted medical textiles include surgical mesh, hernia repair and urogynecologic slings. They are used for implantable and other applications. Braided medical textiles applications include suture, ligament fixation and component attachment. They are manufactured by a combination of different absorbed and permanent fibres. Woven medical textiles are lightweight strength. The common application include as heart-valves, ligament repair and orthopedic spacers. Nonwoven medical textiles are regarded as the most effective material for bacteria barriers. The medical textiles can facilitate and enhance infection prevention and control effort in the healthcare setting thus reducing the problem of cross contamination Non-woven medical textiles can be used to replace and repair different organs and bones. The medical textiles act as an interface between man and treatment in hospitals and other medical centers.

Hospital is a healthcare institution that provides patients treatment by specialized staff and equipment. ${ }^{10}$ Hospitals are usually built by the public, charity organizations, government and individuals. Hospitals have four major categories, thus, acute care community hospitals, specialty/psychiatric hospitals and teaching hospitals. ${ }^{11}$ These hospitals have various departments that use varieties of medical textiles to handle different cases of health conditions and to control and avoid infections in hospitals. One may contract diseases from poorly treated medical textiles. Such nosocomial diseases include scabies, rashes and other pathogenic diseases. Rodrigue noted that contaminated medical textiles transfer pathogens to patients and workers in hospitals especially when the textiles are not properly cared for. ${ }^{12}$ It is observed that patients particularly, patients that have accidents bleed to death in area of study. Chinta and Veena opined that medical textiles protect healthcare givers from infections and help in the control of bleeding in time of accidents. ${ }^{13}$ The purpose of this study was therefore to investigate the health care practices in government hospitals in Enugu State, specifically to; 
I. Determine the availability of medical textiles in government hospitals.

II. Find the maintenance of medical textiles in government hospitals.

The two questions that arise are

I. What are the medical textiles available in government hospitals?

II. How are the medical textiles in government hospitals maintained?

The findings of this study show that medical textiles are not enough in government hospitals in Enugu State. The five identified classes of medical textiles which include knitted medical textiles braided, woven, nonwoven and healthcare and hygiene medical textiles are short in quantity in the area of study. ${ }^{14}$ Study highlighted that the few medical textiles available in the hospitals are not properly maintained. Soiled bedding, door and window curtains among others are not properly laundered and changed in the hospitals. The available, medical textiles are not laundered and sterilized with appropriate equipment like autoclave machines. This condition may encourage transfer of pathogenic organisms very easily to patients and staff in the area of study. Based on the findings of this study, the following recommendations are made;

I. The federal and state governments should map out substantial amount of money for medical textiles every year. This provision may be through ministry of health in every state.

II. The medical supervisors responsible for hospital supervision should ensure regular visit to government hospitals in Enugu State and in the entire hospitals in the federation to make sure that the medical textiles supplied by government are in the hospitals.

III. Maintenance equipment especially laundry equipment such as sterilizing materials like autoclave machines should be provided in every government hospitals in the federation.

IV. There should be training and re-training of orderlies and porters in hospitals on how to care for medical textiles.

\section{Health and safety of textiles using nanomaterials}

Textiles are one of the most heavily traded commodities in the world. The industry is very diverse and its products are used by virtually everybody from private households to large businesses. The textile industry is already an important user of nanotechnologies and there are a significant number of "nanotextiles" in the market, including many consumer goods, with the incorporation of nanoparticles. These include many textiles used in direct contact with the skin, such as underwear, shirts and socks but also interior textiles like cushions, blankets or mattress covers.

There is a knowledge gap between the technological progress in nanotechnology and nanosafety research which is estimated to be 20 years, and it is likely to expand. The European Agency for Safety and Health at Work has established as priority for research related to the safety and health in Europe during the period 2013-2020 the increase of knowledge on nano materials in occupational settings, including new generation nano materials and understand their characteristics in relation to toxicity in biological systems. ${ }^{15}$ The risk for the workers and for the consumers is linked to the characteristic properties of certain nanomaterials that make them different from their macroscale counterparts and will be determined by the chemical composition of the nanomaterial, its physicochemical properties, the interactions with the textile materials and the potential exposure levels. Ingestion exposure via the gut, airborne exposure via the lungs and dermal exposure are the most important exposure routes to be considered in a risk analysis. In addition, the increasing use of nanomaterials, including for industrial purposes, raises specific concerns regarding their disposal at the end of their life cycle with the unavoidable release to the Environment that may lead to indirect human exposure. At present, some EU Regulations already include a specific mention to nanomaterials. This is the case of food (including additives and packaging), biocides and cosmetic products. But this is still not the case of textiles.

The growing concern about the possible negative effects of nanomaterials on humans and on the environment can lead to restrictions to "nanotextiles". In fact, for instance, the 2014 version of the ecological label GOTS (Global Organic Textile Standard) fully bans the presence of nano finishes in textiles. Also in the recent discussion of the new version of the EU ecolabel for textiles, there were several voices to exclude nano materials. In the present paper, after making an overview of the use of nanotechnology in textiles, with special emphasis on textiles for major consumer applications, the safety and health concerns related to nano textiles are presented. The paper includes then a case study concerning the development of a test method to evaluate the skin exposure to nanoparticles, mainly directed to the transfer of the nanoparticles from the textile to the skin. This paper does not deal with the penetration of the nanoparticles into the skin. There are many studies about this topic, related for instance to sunscreens and cosmetics, which are often based on nanomaterials. In fact, only the smaller nanoparticles seem to be able to penetrate in the undamaged skin, although in the skin is injured, larger nanoparticles can penetrate. ${ }^{16}$ In a recent study, ${ }^{17}$ made a literature survey involving 129 relevant publications and concluded that the smaller nanoparticles, with dimensions smaller than $4 \mathrm{~nm}$, can easily penetrate the skin, while those with dimensions from $4 \mathrm{~nm}$ than $20 \mathrm{~nm}$, can potentially penetrate intact skin. Nanoparticles with size between 21 and $45 \mathrm{~nm}$ can also penetrate and permeate in damaged skin. Nanomaterials have the potential to improve the quality of life and to contribute to industrial competitiveness in Europe. However, the new materials may also pose risks to the environment and raise health and safety concerns. The Scientific Committee on Emerging and Newly Identified Health Risks has concluded that, even though nanomaterials are not per se dangerous, there is still scientific uncertainty about the safety of nanomaterials in many aspects and therefore the safety assessment of the substances must be done on a case-by-case basis. In the case of textiles, there are still very few studies on the possible health risks involved with "nanotextiles".

The release of nanoparticles from textiles is particularly relevant when the incorporation is made by fabric finishing. It can occur by different mechanisms. In this paper, the release resulting from skin contact, involving abrasion and sweat, has been analysed more in detail, involving a possible standard text method. The studies made up to now involve silver and titanium dioxide nanoparticles, which are present in the most common nanotextiles in the consumer market. Nevertheless, there are still a lot of discussions on if these nanoparticles can really penetrate into the different skin layers and on the negative effects on human health. ${ }^{18}$ In the case of titanium dioxide, it is very commonly used in sunscreens, the nano particles being deliberately spread over a large surface of the skin. Comparatively, the dermal exposure coming from textiles is much lower, so the relative relevance of skin exposure coming from textiles can be questioned. Also in the 
case of nanosilver, it is used in deodorants, deliberately put on the skin, food packaging or even in toothpastes. Again the relevance of the silver nanoparticles from the textiles to the human body can also be questioned.

\section{Conclusion}

The health care practices relating to medical textiles have been investigated. A sample of 1200 hospital personnel has been studied. Data collected were analyzed using frequency and mean. The findings include that medical textiles are not enough and the few medical textiles available in the government hospitals are not properly maintained. The findings of the study will create awareness to the hospital management in the provision and maintenance of medical textiles. Federal government should sponsor and monitor the provision of medical textiles in the hospitals; there should also be regular supervision by medical supervisors to ensure that the medical textiles supplied in the government hospitals are properly cared for. Nanoparticles can be released from the textile materials due to different effects (abrasion and other mechanical stresses, sweat, irradiation, washing, temperature changes, etc.). It is then expectable that "nanotextiles" may release individual nanoparticles, agglomerates of nanoparticles or small particles of textile with or without nanoparticles, depending on the type of integration of the nanoparticles in textiles. The most important exposure route of the human body to nanoparticles in case of textiles is skin contact. Several standards are being developed under the auspices of the European Committee for Standardization. In this paper, it is presented the development and application of a test method to evaluate the skin exposure to nanoparticles, to evaluate the transfer of the nanoparticles from the textile to the skin by the effect of abrasion and sweat.

\section{Acknowledgements}

None.

\section{Conflict of interest}

The author declares there is no conflict of interest.

\section{References}

1. Martin EA. Meaning of health care. Concise medical. Dictionary Oxford Paperback. 2010.
2. The Institute of medicine. Heaithcare malarial. Peer Point Medical Education, Institute; 2102.

3. Marriam W. Healthcare services in medical treatment. 2013.

4. Savolainen K, Backman U, Brouwer D, et al. L 2013. Nanosafety in Europe 2015-2025: Towards Safe and Sustainable Nanomaterials and Nanotechnology Innovations. Finnish Institute of Occupational Health. 2013. 1-208.

5. Bricher JT. Towards a dynamic definition of health and disease. Med Healthcare Philos. 2005;8(3):335-341.

6. Health Insurance Portability and Accountability Act. Health care services. 2013.

7. Impex AR. Biomedical structures. 2010.

8. Mazharul 1Ic. Introduction of medical textiles/requirement of medical textiles for medical applications. 2012.

9. Impex AR. Biomedical structures. 2010.

10. Santa J. Why linens should be changed in hospital beds. 2013.

11. Glossary of Term. Glossary of term paherts care link. 2014.

12. Rodrigues LV. Medical textiles and apparel. Chemical and textile Division office of industries. Router Bridge. 2012.

13. Chinta SK, Veena KV. Impact of textile on medical field. International Journal of Latest Trends in Engineering and Technology. 2013;3(2):142147.

14. Akubue BN, Anikweze GU. Health Care Practices For Medical Textiles in Government Hospitals. Journal of Education and Practice. 2015;6(26):122.

15. European Agency for Safety and Health at Work. Priorities for occupational safety andhealth research in Europe: 2013-2020. Publications Office of the European Union, Luxembourg. 2013. p.1-107.

16. Labouta H, El-Khordagui L, Krausc T. et al. Mechanism and determinants of nanoparticle penetration through human skin. Nanoscale. 2011;3:4989-4999.

17. Larese Filon F, Mauro M, Adami G, et al. Nanoparticles skin absorption: New aspects for a safety profile evaluation. Regul Toxicol Pharmacol. $2015 ; 72(2): 310-322$.

18. L Almeida, D Ramos. Health and safety concerns of textiles with nanomaterials. IOP Conf Ser Mater Sci Eng. 2017;254:102002. 